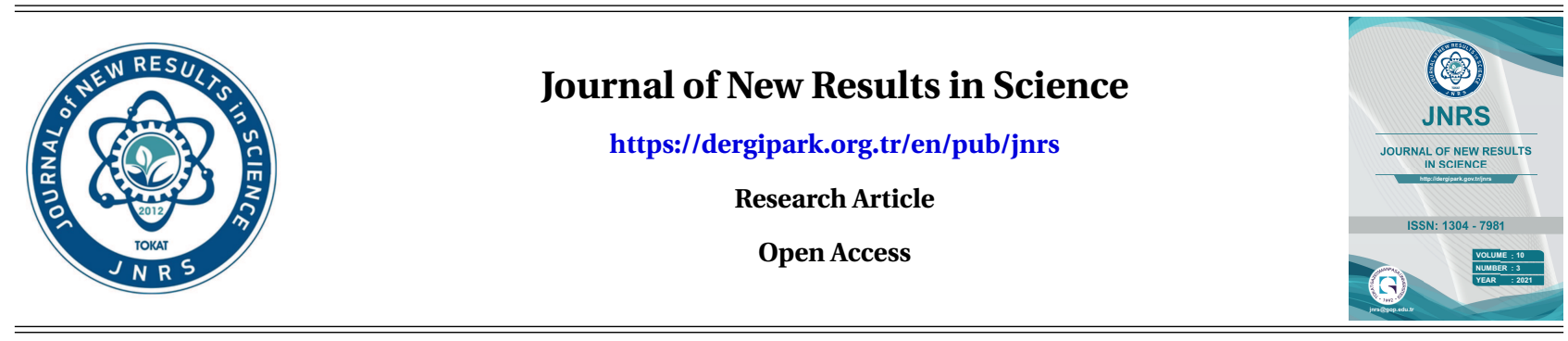

\title{
Properties of invertible elements in complete intuitionistic fuzzy pseudo normed algebra
}

\author{
Bivas Dinda $^{1}(\mathbb{D})$, Santanu Kumar Ghosh $^{2}$ (D) , Tapas Kumar Samanta $^{3}$ (D)
}

\begin{abstract}
Keywords
IFPNA,

Invertible element, Intuitionistic fuzzy

continuity
\end{abstract}

\begin{abstract}
In this paper, we deal with the invertible elements in a complete intuitionistic fuzzy pseudo normed algebra(in short, IFPNA) with respect to Archimedean $t$-norm and Archimedean $t$-conorm. It is done by studying the continuity of algebraic operations in a complete IFPNA and investigating the condition for existence of inverse of an element in a complete IFPNA. Also some properties of invertible elements are studied. It is observed that the set of invertible elements in a complete IFPNA is an open set.
\end{abstract}

Subject Classification (2020): 08A72, 46S40.

\section{Introduction}

For dealing with uncertainty, Zadeh extended the classical set theory and initiated the idea of fuzzy set theory [1]. In 1986, K.T. Atanassov extended the notion of fuzzy set and proposed intuitionistic fuzzy set [2]. Fuzzy norm was first studied by A.K. Katsaras [3, 4]. Thereafter the concept of fuzzy normed spaces were presented in succession by C. Felbin [5] in 1992, S.C. Cheng and J.N. Morderson [6] in 1994, T. Bag and S.K. Samanta [7] in 2003, R. Saadati and S.M. Vaezpour [8] in 2005, I. Golet [9] in 2010 and many other researchers [10-12] in past years.

Fuzzy Banach algebra was first introduced by I. Sadeqi and A. Amiripour [12] in 2007. Dinda et. al. [13] in 2011, proposed a notion of intuitionistic fuzzy Banach algebra. Fuzzy Menger normed algebra was studied by A.K. Mirmostaface [14] in 2012. Following the concept of Mirmostaface [14], Bînzar et. al. [15] in 2016 studied condition for continuous product in fuzzy normed algebra, N. Konwar and P. Debnath [16] in 2018 studied condition for continuous product in intuitionistic fuzzy n-normed algebra. J.R. Kider and A.H. Ali [17] in 2019 proposed some properties of complete fuzzy normed algebra.

In 2016, S. Nădăban [18] introduced fuzzy pseudo norm and showed that fuzzy pseudo norm is a generalization of fuzzy norm. Following the works S. Nădăban [18] and Bag-Samanta [7, 19], Dinda et. al. [20-23] extended the concept of intuitionistic fuzzy norm and developed intuitionistic fuzzy pseudo norm.

\footnotetext{
${ }^{1}$ rs.bivas@knu.ac.in (Corresponding Author); ${ }^{2}$ santanu_96@yahoo.co.in; ${ }^{3}$ mumpu_tapas5@yahoo.co.in

${ }^{1,2}$ Department of Mathematics, Kazi Nazrul University, Asansol, WB 713340, India.

${ }^{3}$ Department of Mathematics, Uluberia College, Uluberia, WB 711315, India.

Article History: Received: 07 Aug 2021 - Accepted: 29 Nov 2021 - Published: 31 Dec 2021
} 
While solving differential equation, linear algebraic equation, or integral equation, in intuitionistic fuzzy environment there is a problem related to inverse operation. In this paper, we investigate the inverse of an element and properties of the set of invertible elements of a complete IFPNA with respect to Archimedean $t$-norm and Archimedean $t$-conorm.

The structure of this paper is as follows: In section 2, we give some preliminary results which were known before and used in the subsequent sections. In section 3, we propose the definition of intuitionistic fuzzy pseudo normed algebra (IFPNA) with respect to Archimedean $t$-norm and Archimedean $t$-conorm and prove that the algebraic operations are continuous in a complete IFPNA. We also prove a result giving the condition for the existence of the inverse of an element in a complete IFPNA. In section 4 , we show that the set of all invertible elements (I) is an open set and the map $a \rightarrow a^{-1}$ from $I$ into $I$ is strongly intuitionistic fuzzy continuous(IFC).

\section{Preliminaries}

Definition 2.1. [24] A function $*:[0,1] \times[0,1] \rightarrow[0,1]$ is called a $t$-norm if and only if (iff) the following four conditions are satisfied:
(1.1) $e_{1} * e_{2}=e_{2} * e_{1}, \forall e_{1}, e_{2} \in[0,1]$
(1.2) $e_{1} * e_{2} \leq e_{1} * e_{3}$ if $e_{2} \leq e_{3}, \forall e_{1}, e_{2}, e_{3} \in[0,1]$,
(1.3) $e_{1} *\left(e_{2} * e_{3}\right)=\left(e_{1} * e_{2}\right) * e_{3}, \forall e_{1}, e_{2}, e_{3} \in[0,1]$,
(1.4) $e_{1} * 1=e_{1}, \forall e_{1} \in[0,1]$.

Definition 2.2. $[24,25]$ A function $\diamond:[0,1] \times[0,1] \rightarrow[0,1]$ is called a $t$-conorm iff the following four conditions are satisfied:
(2.1) $e_{1} \diamond e_{2}=e_{2} \diamond e_{1}, \forall e_{1}, e_{2} \in[0,1]$,
(2.2) $e_{1} \diamond e_{2} \leq e_{1} \diamond e_{3}$ if $e_{2} \leq e_{3}, \forall e_{1}, e_{2}, e_{3} \in[0,1]$,
(2.3) $e_{1} \diamond\left(e_{2} \diamond e_{3}\right)=\left(e_{1} \diamond e_{2}\right) \diamond e_{3}, \forall e_{1}, e_{2}, e_{3} \in[0,1]$,
(2.4) $e_{1} \diamond 0=e_{1}, \forall e_{1} \in[0,1]$.

Definition 2.3. [25] A $t$-norm $*$ is called Archimedean iff

(1.5) $*$ is continuous, and

(1.6) $e_{1} * e_{1}<e_{1}, \forall e_{1} \in(0,1)$.

Definition 2.4. [25] A $t$-conorm $\diamond$ is called Archimedean iff

$(2.5) \diamond$ is continuous, and

(2.6) $e_{1} \diamond e_{1}>e_{1}, \forall e_{1} \in(0,1)$.

In the subsequent theorems, we use $*$ as Archimedean $t$-norm and $\diamond$ as Archimedean $t$-conorm.

Definition 2.5. [20] Let $V$ be linear space over the field $\mathbb{K}(=\mathbb{R}$ or $\mathbb{C})$. An intuitionistic fuzzy subset $(\mu, v)$ of $(V \times \mathbb{R}, V \times \mathbb{R})$ is said to be an intuitionistic fuzzy pseudo norm on $V$ if $\forall v_{1}, v_{2} \in V$

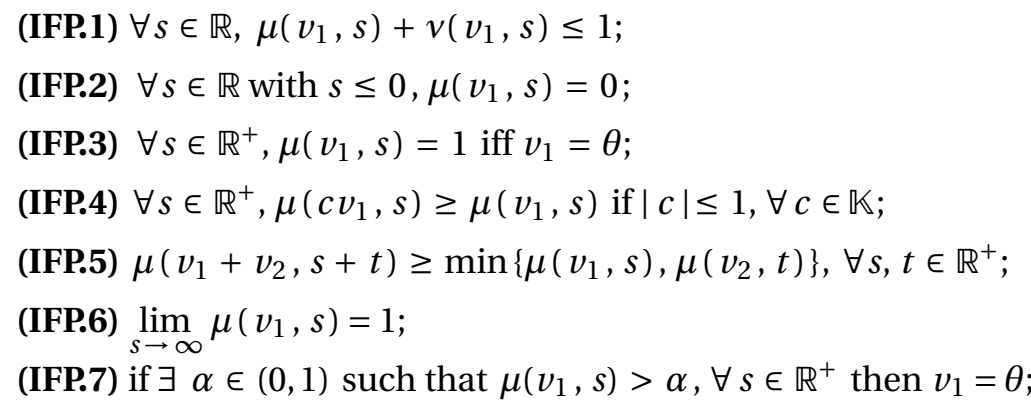


(IFP.8) $\forall v_{1} \in V, \mu\left(\nu_{1}, \cdot\right)$ is left continuous on $\mathbb{R}$;

(IFP.9) $\forall s \in \mathbb{R}$ with $s \leq 0, v\left(\nu_{1}, s\right)=1$;

(IFP.10) $\forall s \in \mathbb{R}^{+}, v\left(\nu_{1}, s\right)=0$ iff $\nu_{1}=\theta$;

(IFP.11) $\forall s \in \mathbb{R}^{+}, v\left(c v_{1}, s\right) \leq v\left(v_{1}, s\right)$ if $|c| \leq 1, \forall c \in \mathbb{K}$;

(IFP.12) $v\left(v_{1}+v_{2}, s+t\right) \leq \max \left\{v\left(v_{1}, s\right), v\left(v_{2}, t\right)\right\}, \forall s, t \in \mathbb{R}^{+}$;

(IFP.13) $\lim _{s \rightarrow \infty} v\left(v_{1}, s\right)=0$;

(IFP.14) if $\exists \alpha \in(0,1)$ such that $v\left(v_{1}, s\right)<\alpha, \forall s \in \mathbb{R}^{+}$then $v_{1}=\theta$;

(IFP.15) $\forall v_{1} \in V, v\left(v_{1}, \cdot\right)$ is left continuous on $\mathbb{R}$.

Here $(V, \mu, v)$ is called intuitionistic fuzzy pseudo normed linear space (IFPNLS).

Definition 2.6. [22] Let $\left(V, \mu_{1}, v_{1}\right),\left(U, \mu_{2}, v_{2}\right)$ be two IFPNLS. A mapping $P:\left(V, \mu_{1}, v_{1}\right) \rightarrow\left(U, \mu_{2}, v_{2}\right)$ is said to be strongly IFC at $v_{0} \in V$ if for any given $\epsilon>0$ there exists $\delta(\epsilon)>0$ such that for all $v \in V$,

$$
\mu_{2}\left(P(\nu)-P\left(v_{0}\right), \epsilon\right) \geq \mu_{1}\left(\nu-v_{0}, \delta\right), v_{2}\left(P(v)-P\left(v_{0}\right), \epsilon\right) \leq v_{1}\left(\nu-v_{0}, \delta\right) .
$$

Definition 2.7. [21] Let $(V, \mu, v)$ be an IFPNLS. An open ball $B(v, r, s)$ with center at $x$, radius $0<r<1$ and $s \in \mathbb{R}^{+}$is defined by

$$
B(\nu, r, s)=\{a \in V: \mu(\nu-a, s)>1-r, v(\nu-a, s)<r\} .
$$

\section{Condition for existence of invertible element in complete IFPNA}

Throughout this paper unless otherwise stated we take $*$ as Archimedean $t$-norm and $\diamond$ as Archimedean $t$-conorm. In this section we endeavor to investigate the condition for existence of an invertible element in a complete IFPNA.

Definition 3.1. Let $V$ be an algebra over the field of complex numbers, $*$ be the Archimedean t-norm and $\diamond$ be the Archimedean t-conorm. By an intuitionistic fuzzy pseudo normed algebra(in short, IFPNA) we mean an intuitionistic fuzzy subset $(\mu, v)$ of $\left(V \times \mathbb{R}^{+}, V \times \mathbb{R}^{+}\right)$such that

(IFPNA.1) $(V, \mu, v)$ is an IFPNLS.

(IFPNA.2) $\mu\left(v_{1} v_{2}, s t\right) \geq \mu\left(v_{1}, s\right) \diamond \mu\left(v_{2}, t\right), v\left(v_{1} v_{2}, s t\right) \leq v\left(v_{1}, s\right) * v\left(v_{2}, t\right), \forall v_{1} v_{2} \in V$, and for each $s, t \in \mathbb{R}^{+}$.

If $(V, \mu, v)$ be a complete IFPNLS then the normed algebra $(V, \mu, v, *, \diamond)$ will be complete IFPNA.

Example 3.2. Let $(V,\|\cdot\|)$ be a pseudo normed algebra, and consider $a \diamond b=\max \{a, b\}$ and $a * b=\min \{a, b\}$.

Define $\mu, v: V \times \mathbb{R} \rightarrow[0,1]$ by

$$
\begin{aligned}
& \mu(\nu, s)= \begin{cases}\frac{s}{s+\|v\|} & \text { if } s>\|v\|, \\
0 & \text { if } s \leq\|v\| .\end{cases} \\
& v(v, s)= \begin{cases}\frac{\|v\|}{s+\|v\|} & \text { if } s>\|v\|, \\
1 & \text { if } s \leq\|v\| .\end{cases}
\end{aligned}
$$

We will show that $(V, \mu, v, \diamond, *)$ is an IFPNA on $V$.

From the Example 3.2 of [20] it is easy to verify the conditions of (IFPNA.1). Now we show (IFPNA.2).

Clearly $*=\min$, and $\diamond=\max$ are Archimedean $t$-norm and Archimedean $t$-conorm.

Let $v_{1}, v_{2} \in V$ and $s \in \mathbb{R}^{+}$. Without loss of generality let us assume $\mu\left(v_{1}, s\right) \geq \mu\left(v_{2}, s\right)$. Then $\left\|v_{1}\right\| \leq\left\|v_{2}\right\|$. Now 
we have the following cases for consideration.

Case-1:

$$
\begin{aligned}
s>\left\|v_{2}\right\| \geq\left\|v_{1}\right\| & \Rightarrow s\left\|v_{1}\right\|>\left\|v_{1}\right\|\left\|v_{2}\right\| \geq\left\|v_{1} v_{2}\right\| \\
& \Rightarrow s^{2}\left\|v_{1}\right\|>s\left\|v_{1} v_{2}\right\| \\
& \Rightarrow s^{3}+s^{2}\left\|v_{1}\right\|>s^{3}+s\left\|v_{1} v_{2}\right\| \\
& \Rightarrow s^{2}\left(s+\left\|v_{1}\right\|\right)>s\left(s^{2}+\left\|v_{1} v_{2}\right\|\right) \\
& \Rightarrow \frac{s^{2}}{s^{2}+\left\|\nu_{1} v_{2}\right\|}>\frac{s}{s+\left\|v_{1}\right\|} \\
& \Rightarrow \mu\left(v_{1} v_{2}, s^{2}\right)>\max \left\{\mu\left(v_{1}, s\right), \mu\left(v_{2}, s\right)\right\} .
\end{aligned}
$$

Case-2:

$$
\begin{aligned}
s \leq\left\|v_{1}\right\| \leq\left\|v_{2}\right\| & \Rightarrow \mu\left(v_{1}, s\right)=0, \mu\left(v_{2}, s\right)=0 \\
& \Rightarrow \mu\left(v_{1} v_{2}, s^{2}\right) \geq \max \left\{\mu\left(v_{1}, s\right), \mu\left(v_{2}, s\right)\right\} .
\end{aligned}
$$

Also, Without loss of generality let us assume $v\left(\nu_{1}, s\right) \geq v\left(v_{2}, s\right)$. Now we have the following cases.

Case-1:

$$
\begin{aligned}
s>\left\|v_{2}\right\| \geq\left\|v_{1}\right\| & \Rightarrow s\left\|v_{2}\right\|>\left\|v_{1}\right\|\left\|v_{2}\right\| \geq\left\|v_{1} v_{2}\right\| \\
& \Rightarrow s^{2}\left\|v_{2}\right\|>s\left\|v_{1} v_{2}\right\| \\
& \Rightarrow s^{2}\left\|v_{2}\right\|+\left\|v_{2}\right\|\left\|v_{1} v_{2}\right\|>s\left\|v_{1} v_{2}\right\|+\left\|v_{2}\right\|\left\|v_{1} v_{2}\right\| \\
& \Rightarrow\left\|v_{2}\right\|\left(s^{2}+\left\|v_{1} v_{2}\right\|\right)>\left\|v_{1} v_{2}\right\|\left(s+\left\|v_{2}\right\|\right) \\
& \Rightarrow \frac{\left\|v_{1} v_{2}\right\|}{s^{2}+\left\|v_{1} v_{2}\right\|}<\frac{\left\|v_{2}\right\|}{s+\left\|v_{2}\right\|} \\
& \Rightarrow v\left(v_{1} v_{2}, s^{2}\right)<\min \left\{v\left(v_{1}, s\right), v\left(v_{2}, s\right)\right\} .
\end{aligned}
$$

Case-2:

$$
\begin{aligned}
s \leq\left\|v_{1}\right\| \leq\left\|v_{2}\right\| & \Rightarrow v\left(v_{2}, s\right)=1, v\left(v_{1}, s\right)=1 \\
& \Rightarrow v\left(v_{1} v_{2}, s^{2}\right) \leq \min \left\{v\left(v_{1}, s\right), v\left(v_{2}, s\right)\right\} .
\end{aligned}
$$

Therefore, (IFPNA.2) is satisfied and hence $(V, \mu, v, \diamond, *)$ is an IFPNA.

Example 3.3. Let $(V,\|\cdot\|)$ be a pseudo normed linear space. Define $\mu, v: V \times \mathbb{R} \rightarrow[0,1]$ by

$$
\begin{aligned}
& \mu(v, s)= \begin{cases}1 & \text { if } s>\|v\|, \\
0 & \text { if } s \leq\|v\| .\end{cases} \\
& v(v, s)= \begin{cases}0 & \text { if } s>\|v\|, \\
1 & \text { if } s \leq\|v\| .\end{cases}
\end{aligned}
$$

Then $(V, \mu, v, \diamond, *)$ is an IFPNA on $V$. 
From the Example 3.1 of [20] and from Example 3.2, the proof follows.

Theorem 3.4. In an IFPNA $(V, \mu, v, \diamond, *)$, the algebraic operations " + " and " • " are continuous.

\section{Proof.}

Let $\left\{a_{n}\right\}_{n}$ and $\left\{b_{n}\right\}_{n}$ be two sequences in $V$ converges to $a$ and $b$ respectively. Then for every $s \in \mathbb{R}^{+}$, $\lim _{n \rightarrow \infty} \mu\left(a_{n}-a, s\right)=1, \lim _{n \rightarrow \infty} v\left(a_{n}-a, s\right)=0$ and $\lim _{n \rightarrow \infty} \mu\left(b_{n}-b, s\right)=1, \lim _{n \rightarrow \infty} v\left(b_{n}-b, s\right)=0$. Now, $\mu\left(\left(a_{n}+b_{n}\right)-(a+b), s\right) \geq \min \left\{\mu\left(a_{n}-a, \frac{s}{2}\right), \mu\left(b_{n}-b, \frac{s}{2}\right)\right\}$. Hence

$$
\begin{aligned}
& \lim _{n \rightarrow \infty} \mu\left(\left(a_{n}+b_{n}\right)-(a+b), s\right) \\
& \geq \min \left\{\lim _{n \rightarrow \infty} \mu\left(a_{n}-a, \frac{s}{2}\right), \lim _{n \rightarrow \infty} \mu\left(b_{n}-b, \frac{s}{2}\right)\right\} \\
& \geq \min \{1,1\}=1 .
\end{aligned}
$$

This proves the continuity of addition in $(V, \mu, v, \diamond, *)$.

$$
\text { Again, } \begin{aligned}
\mu\left(a_{n} b_{n}-a b, s\right) & =\mu\left(a_{n} b_{n}-a_{n} b+a_{n} b-a b, s\right) \\
& =\mu\left(a_{n}\left(b_{n}-b\right)+b\left(a_{n}-a\right), s\right) \\
& \geq \min \left\{\mu\left(a_{n}\left(b_{n}-b\right), \frac{s}{2}\right), \mu\left(b\left(a_{n}-a\right), \frac{s}{2}\right)\right\} \\
& \geq \min \left\{\mu\left(a_{n}, \sqrt{\frac{s}{2}}\right) \diamond \mu\left(b_{n}-b, \sqrt{\frac{s}{2}}\right), \mu\left(b, \sqrt{\frac{s}{2}}\right) \diamond \mu\left(a_{n}-a, \sqrt{\frac{s}{2}}\right)\right\} .
\end{aligned}
$$

Therefore, $\lim _{n \rightarrow \infty} \mu\left(a_{n} b_{n}-a b, s\right)$

$$
\begin{aligned}
& \geq \min \left\{\lim _{n \rightarrow \infty} \mu\left(a_{n}, \sqrt{\frac{s}{2}}\right) \diamond 1, \lim _{n \rightarrow \infty} \mu\left(b, \sqrt{\frac{s}{2}}\right) \diamond 1\right\} \\
& \geq \min \{1,1\} \\
& =1 .
\end{aligned}
$$

Hence multiplication is also continuous in $(V, \mu, v, \diamond, *)$.

Theorem 3.5. Let $(V, \mu, v, \diamond, *)$ be a complete IFPNA. If $a \in B(0, r, s), s \in \mathbb{R}^{+}$and $0<r<1$ then the inverse of $(i-a)$ exists and

$$
(i-a)^{-1}=i+\sum_{n=1}^{\infty} a^{n}
$$

where $i$ and 0 are the identity and the zero of $V$ respectively.

\section{Proof.}

Since $a \in B(0, r, s)$ we have $\mu(a, s)>1-r, v(a, s)<r$, for each $s \in \mathbb{R}^{+}$and $0<r<1$.

First we verify the convergence of the series $\sum_{n=1}^{\infty} a^{n}$ in $V$. 
Let $u_{n}=\sum_{j=1}^{n} a^{j}$ then it is enough to verify that $\mu\left(u_{n+p}-u_{n}, s\right)>1-r$ and $v\left(u_{n+p}-u_{n}, s\right)<r$. Now,

$$
\begin{aligned}
& \mu\left(u_{n+p}-u_{n}, s\right) \\
& =\mu\left(a^{n+1}+a^{n+2}+\cdots+a^{n+p}, s\right) \\
& \geq \min \left\{\mu\left(a^{n+1}, \frac{s}{p}\right), \mu\left(a^{n+2}, \frac{s}{p}\right), \cdots, \mu\left(a^{n+p}, \frac{s}{p}\right)\right\} \\
& \geq \min \left\{\mu\left(a, \sqrt[n+1]{\frac{s}{p}}\right) \diamond \mu\left(a, \sqrt[n+1]{\frac{s}{p}}\right) \diamond \cdots \diamond \mu\left(a, \sqrt[n+1]{\frac{s}{p}}\right), \mu\left(a, \sqrt[n+2]{\frac{s}{p}}\right) \diamond \mu\left(a, \sqrt[n+2]{\frac{s}{p}}\right) \diamond \cdots \diamond \mu\left(a, \sqrt[n+2]{\frac{s}{p}}\right),\right. \\
& \left.\cdots, \mu\left(a, \sqrt[n+p]{\frac{s}{p}}\right) \diamond \mu\left(a, \sqrt[n+p]{\frac{s}{p}}\right) \diamond \cdots \diamond \mu\left(a, \sqrt[n+p]{\frac{s}{p}}\right)\right\} \\
& >\min \{(1-r) \diamond(1-r) \diamond \cdots \diamond(1-r),(1-r) \diamond(1-r) \diamond \cdots \diamond(1-r), \cdots,(1-r) \diamond(1-r) \diamond \cdots \diamond(1-r)\} \\
& >\min \{(1-r),(1-r), \cdots,(1-r)\},[\text { By }(2.6) \text { of Definition 2.4] } \\
& =1-r \text {. }
\end{aligned}
$$

$$
\begin{aligned}
& v\left(u_{n+p}-u_{n}, s\right) \\
& =v\left(a^{n+1}+a^{n+2}+\cdots+a^{n+p}, s\right) \\
& \leq \max \left\{v\left(a^{n+1}, \frac{s}{p}\right), v\left(a+n, \frac{s}{p}\right), \cdots, v\left(a^{n+p}, \frac{s}{p}\right)\right\} \\
& \leq \max \left\{v\left(a, \sqrt[n+1]{\frac{s}{p}}\right) * v\left(a, \sqrt[n+1]{\frac{s}{p}}\right) * \cdots * v\left(a, \sqrt[n+1]{\frac{s}{p}}\right), v\left(a, \sqrt[n+2]{\frac{s}{p}}\right) * v\left(a, \sqrt[n+2]{\frac{s}{p}}\right) * \cdots * v\left(a, \sqrt[n+2]{\frac{s}{p}}\right),\right. \\
& \left.\cdots, v\left(a, \sqrt[n+p]{\frac{s}{p}}\right) * v\left(a, \sqrt[n+p]{\frac{s}{p}}\right) * \cdots * v\left(a, \sqrt[n+p]{\frac{s}{p}}\right)\right\} \\
& <\max \{r * r * \cdots * r, r * r * \cdots * r, \cdots, r * r * \cdots * r\} \\
& <\max \{r, r, \cdots, r\},[\text { By (1.6) of Definition 2.3] } \\
& =r .
\end{aligned}
$$

Hence the series $\sum_{n=1}^{\infty} a^{n}$ is convergent in $V$.

Now $(i-a)\left(i+a+a^{2}+\cdots+a^{n}\right)=\left(i+a+a^{2}+\cdots+a^{n}\right)(i-a)=i-a^{n+1}$.

Also,

$$
\begin{aligned}
\mu\left(a^{n+1}, s\right) & \geq \mu(a, \sqrt[n+1]{s}) \diamond \mu(a, \sqrt[n+1]{s}) \diamond \cdots \diamond \mu(a, \sqrt[n+1]{s}) \\
& >(1-r) \diamond(1-r) \diamond \cdots \diamond(1-r) \\
& >(1-r),[\text { By (2.6) of Definition 2.4]. } \\
v\left(a^{n+1}, t\right) & \leq v(a, \sqrt[n+1]{s}) * v(a, \sqrt[n+1]{s}) * \cdots * v(a, \sqrt[n+1]{s}) \\
& <r * r * \cdots * r \\
& <r,[\text { By (1.6) of Definition 2.3]. }
\end{aligned}
$$


Hence $a^{n+1}$ converges to 0 as $n \rightarrow \infty$. Therefore taking $n \rightarrow \infty$ we obtain

$$
(i-a)\left(i+\sum_{n=1}^{\infty} a^{n}\right)=\left(i+\sum_{n=1}^{\infty} a^{n}\right)(i-a)=e
$$

Thus $(i-a)^{-1}=i+\sum_{n=1}^{\infty} a^{n}$.

Corollary 3.6. Let $(V, \mu, v, \diamond, *)$ be a complete IFPNA. If $i-a \in B(0, r, s)$, for each $s \in \mathbb{R}^{+}$and $0<r<1$ then the inverse of $a$ exists and

$$
a^{-1}=i+\sum_{n=1}^{\infty}(i-a)^{n}
$$

Corollary 3.7. Let $(V, \mu, v, \diamond, *)$ be a complete IFPNA. If $\lambda(\neq 0)$ be a scalar such that $\frac{a}{\lambda} \in B(0, r, s)$, for each $s \in \mathbb{R}^{+}$and $0<r<1$ then the inverse of $(\lambda i-a)$ exists and

$$
(\lambda i-a)^{-1}=\sum_{n=1}^{\infty} \lambda^{-n} a^{n-1}
$$

\section{Proof.}

Since $\frac{a}{\lambda} \in B(0, r, s)$ for each $s \in \mathbb{R}^{+}$and $0<r<1$, then

$$
\mu\left(\frac{a}{\lambda}, s\right)>1-r, v\left(\frac{a}{\lambda}, s\right)<r \Rightarrow \mu\left(i-\left(i-\frac{a}{\lambda}\right), s\right)>1-r, v\left(i-\left(i-\frac{a}{\lambda}\right), s\right)<r
$$

Hence by Corollary 3.6, the inverse of $\left(i-\frac{a}{\lambda}\right)$ exists i.e., the inverse of $(\lambda i-a)$ exists.

$$
\text { Also, } \begin{aligned}
(\lambda i-a)^{-1} & =\lambda^{-1}\left(i-\lambda^{-1} a\right)^{-1} \\
& =\lambda^{-1}\left[i+\sum_{n=1}^{\infty}\left\{i-\left(i-\lambda^{-1} a\right)\right\}^{n}\right], \text { [by Corollary 3.6] } \\
& =\lambda^{-1}\left[i+\sum_{n=1}^{\infty} \lambda^{-n} a^{n}\right] \\
& =\sum_{n=1}^{\infty} \lambda^{-n} a^{n-1}
\end{aligned}
$$

\section{Properties of invertible elements in IFPNA}

Theorem 4.1. Let $(V, \mu, v, \diamond, *)$ be a complete IFPNA. Then set of invertible elements of $V$ is an open set.

\section{Proof.}

Let $I$ be the set of invertible elements of $V$. Let $a_{0} \in I$, then we have to show that $B\left(a_{0}, r, s\right) \subseteq I$ for each $s \in \mathbb{R}^{+}$with $0<r<1$.

Let $a \in B\left(a_{0}, r, s\right)$ then $\mu\left(a-a_{0}, s\right)>1-r$ and $v\left(a-a_{0}, s\right)<r$.

Let us take $r \in(0,1)$ such that $r<\min \left\{1-\mu\left(a_{0}^{-1}, s\right), v\left(a_{0}^{-1}, s\right)\right\}$. Then

$$
\mu\left(a-a_{0}, s\right)>1-r>\mu\left(a_{0}^{-1}, s\right), v\left(a-a_{0}, s\right)<r<v\left(a_{0}^{-1}, s\right) .
$$

Let $b=a_{0}^{-1} a$, and $c=i-b$, where $i$ is the identity of $V$. Then for all $s \in \mathbb{R}^{+}$,

$\mu(c, s)=\mu(i-b, s)$

$=\mu(b-i, s),[$ by (IFP.4)] 


$$
\begin{aligned}
& =\mu\left(a_{0}^{-1} a-a_{0}^{-1} a_{0}, s\right) \\
& =\mu\left(a_{0}^{-1}\left(a-a_{0}\right), s\right) \\
& \geq \mu\left(a_{0}^{-1}, \sqrt{s}\right) \diamond \mu\left(a-a_{0}, \sqrt{s}\right),[\text { by (IFPNA.2)] } \\
& >\mu\left(a-a_{0}, \sqrt{s}\right) \diamond \mu\left(a-a_{0}, \sqrt{s}\right),[\text { by Equation } 4.1, \text { and }(2.2) \text { of Definition 2.2] } \\
& >\mu\left(a-a_{0}, \sqrt{s}\right),[\text { by }(2.6) \text { of Definition } 2.4] \\
& >1-r, \text { since } a \in B\left(a_{0}, r, s\right) \text { for all } s \in \mathbb{R}^{+} \text {with } 0<r<1 . \\
v(c, s) & =v(i-b, s) \\
& =v(b-i, s),[\text { by }(\text { IFP.11)] } \\
& =v\left(a_{0}^{-1} a-a_{0}^{-1} a_{0}, s\right) \\
& =v\left(a_{0}^{-1}\left(a-a_{0}\right), s\right) \\
& \leq v\left(a_{0}^{-1}, \sqrt{s}\right) * v\left(a-a_{0}, \sqrt{s}\right),[\text { by (IFPNA.2)] } \\
& <v\left(a-a_{0}, \sqrt{s}\right) * v\left(a-a_{0}, \sqrt{s}\right),[\text { by Equation } 4.1, \text { and }(1.2) \text { of Definition 2.1] } \\
& <v\left(a-a_{0}, \sqrt{s}\right),[\text { by }(1.6) \text { of Definition } 2.3] \\
& <r, \text { since } a \in B\left(a_{0}, r, s\right) \text { for all } s \in \mathbb{R}^{+} \text {with } 0<r<1 .
\end{aligned}
$$

Hence $c \in B(0, r, s)$ and therefore by Theorem 3.5, $(i-c)$ is invertible; i.e., $b$ is invertible. Hence $b \in I$. Now $a_{0} \in I$ and $b \in I$. Hence $a_{0} . b \in I$; i.e., $a_{0} a_{0}^{-1} a \in I$; i.e., $a \in I$.

Thus $B\left(a_{0}, r, s\right) \subseteq I$ and hence $I$ is an open in $V$.

Corollary 4.2. Let $(V, \mu, v, \diamond, *)$ be a complete IFPNA. Then set of non-invertible elements of $V$ is a closed set.

Theorem 4.3. Let $I$ be the set of invertible elements of a complete IFPNA $(V, \mu, v, \diamond, *)$. Then the mapping $P$ from $a$ to $a^{-1}$ of $I$ into $I$ is strongly IFC.

\section{Proof.}

Let $a_{0} \in I$, then by Theorem 4.1, $B\left(a_{0}, r, s\right) \subseteq I$, where $s \in \mathbb{R}^{+}$and $0<r<1$.

Let $r<\min \left\{1-\mu\left(a_{0}^{-1}, t\right), v\left(a_{0}^{-1}, t\right)\right\}$, and $a \in I$ be such that $a \in B\left(a_{0}, r, s\right)$. Then we have the Equation 4.1.

Now by Corollary 3.6, $a^{-1} a_{0}=\left(a_{0}^{-1} a\right)^{-1}=i+\sum_{n=1}^{\infty}\left(i-a_{0}^{-1} a\right)^{n}$. Therefore,

$$
\begin{aligned}
& \mu\left(a^{-1} a_{0}-i, \frac{\pi^{2}}{6}\right) \\
& =\mu\left(\sum_{n=1}^{\infty}\left(i-a_{0}^{-1} a\right)^{n}, 1+\frac{1}{4}+\frac{1}{9}+\frac{1}{16}+\cdots\right) \\
& \geq \min \left\{\mu\left(\left(i-a_{0}^{-1} a\right), 1\right), \mu\left(\left(i-a_{0}^{-1} a\right)^{2}, \frac{1}{4}\right), \cdots, \mu\left(\left(i-a_{0}^{-1} a\right)^{n}, \frac{1}{n^{2}}\right), \cdots\right\}, \quad[\text { by (IFP.5)] } \\
& \geq \min \left\{\mu\left(a_{0}^{-1}\left(a_{0}-a\right), 1\right), \mu\left(a_{0}^{-1}\left(a_{0}-a\right), \frac{1}{2}\right) \diamond \mu\left(a_{0}^{-1}\left(a_{0}-a\right), \frac{1}{2}\right), \cdots\right\},[\text { by (IFPNA.2)] } \\
& \geq \min \left\{\mu\left(a_{0}^{-1}, 1\right) \diamond \mu\left(\left(a_{0}-a\right), 1\right), \mu\left(a_{0}^{-1}, \frac{1}{\sqrt{2}}\right) \diamond \mu\left(\left(a_{0}-a\right), \frac{1}{\sqrt{2}}\right) \diamond \mu\left(a_{0}^{-1}, \frac{1}{\sqrt{2}}\right) \diamond \mu\left(\left(a_{0}-a\right), \frac{1}{\sqrt{2}}\right), \cdots\right\}, \quad[\text { by (IFPNA.2)] } \\
& >\min \left\{\mu\left(\left(a_{0}-a\right), 1\right) \diamond \mu\left(\left(a_{0}-a\right), 1\right), \mu\left(\left(a_{0}-a\right), \frac{1}{\sqrt{2}}\right) \diamond \mu\left(\left(a_{0}-a\right), \frac{1}{\sqrt{2}}\right) \diamond \mu\left(\left(a_{0}-a\right), \frac{1}{\sqrt{2}}\right) \diamond \mu\left(\left(a_{0}-a\right), \frac{1}{\sqrt{2}}\right), \cdots\right\} \\
& >\min \left\{\mu\left(\left(a_{0}-a\right), 1\right), \mu\left(\left(a_{0}-a\right), \frac{1}{\sqrt{2}}\right), \cdots\right\},[\text { by }(2.6) \text { of Definition } 2.4] \\
& \geq \mu\left(\left(a_{0}-a\right), \lim _{n \rightarrow \infty}\left(\frac{1}{n^{2}}\right)^{\frac{1}{2 n}}\right), \text { since } \mu(a, \cdot) \text { is a non decreasing function and } 1>\frac{1}{4^{\frac{1}{4}}}>\frac{1}{9^{\frac{1}{6}}}>\frac{1}{16^{\frac{1}{8}}}>\cdots>\frac{1}{n^{2 \frac{1}{2 n}}}>\cdots
\end{aligned}
$$


$=\mu\left(\left(a_{0}-a\right), \lim _{n \rightarrow \infty} \frac{1}{n^{\frac{1}{n}}}\right)$

$=\mu\left(\left(a_{0}-a\right), 1\right)$

$\geq \mu\left(a-a_{0}, 1\right)$, [by (IFP.4)].

Hence

$$
\mu\left(a^{-1} a_{0}-i, \frac{\pi^{2}}{6}\right)>\mu\left(\left(a-a_{0}\right), 1\right)
$$

Also,

$$
\begin{aligned}
& v\left(a^{-1} a_{0}-e, \frac{\pi^{2}}{6}\right) \\
& =v\left(\sum_{n=1}^{\infty}\left(i-a_{0}^{-1} a\right)^{n}, 1+\frac{1}{4}+\frac{1}{9}+\frac{1}{16}+\cdots\right) \\
& \leq \max \left\{v\left(\left(i-a_{0}^{-1} a\right), 1\right), v\left(\left(i-a_{0}^{-1} a\right)^{2}, \frac{1}{4}\right), \cdots, v\left(\left(i-a_{0}^{-1} a\right)^{n}, \frac{1}{n^{2}}\right), \cdots\right\},[\mathrm{by} \text { (IFP.12)] } \\
& \leq \max \left\{v\left(a_{0}^{-1}\left(a_{0}-a\right), 1\right), v\left(a_{0}^{-1}\left(a_{0}-a\right), \frac{1}{2}\right) * v\left(a_{0}^{-1}\left(a_{0}-a\right), \frac{1}{2}\right), \cdots\right\},[\mathrm{by} \text { (IFPNA.2)] } \\
& \leq \max \left\{v\left(a_{0}^{-1}, 1\right) * v\left(\left(a_{0}-a\right), 1\right), v\left(a_{0}^{-1}, \frac{1}{\sqrt{2}}\right) * v\left(\left(a_{0}-a\right), \frac{1}{\sqrt{2}}\right) * v\left(a_{0}^{-1}, \frac{1}{\sqrt{2}}\right) * v\left(\left(a_{0}-a\right), \frac{1}{\sqrt{2}}\right), \cdots\right\},[\mathrm{by} \text { (IFPNA.2)] } \\
& <\max \left\{v\left(\left(a_{0}-a\right), 1\right) * v\left(\left(a_{0}-a\right), 1\right), v\left(\left(a_{0}-a\right), \frac{1}{\sqrt{2}}\right) * v\left(\left(a_{0}-a\right), \frac{1}{\sqrt{2}}\right) * v\left(\left(a_{0}-a\right), \frac{1}{\sqrt{2}}\right) * v\left(\left(a_{0}-a\right), \frac{1}{\sqrt{2}}\right), \cdots\right\}
\end{aligned}
$$

[by Equation 4.1 and (1.2) of Definition 2.1]

$<\max \left\{v\left(\left(a_{0}-a\right), 1\right), v\left(\left(a_{0}-a\right), \frac{1}{\sqrt{2}}\right), \cdots\right\}, \quad$ by (1.6) of Definition 2.3]

$\leq v\left(\left(a_{0}-a\right), \lim _{n \rightarrow \infty}\left(\frac{1}{n^{2}}\right)^{\frac{1}{2 n}}\right)$, since $v(a, \cdot)$ is a non increasing function and $1>\frac{1}{4^{\frac{1}{4}}}>\frac{1}{9^{\frac{1}{6}}}>\frac{1}{16^{\frac{1}{8}}}>\cdots>\frac{1}{n^{\frac{1}{2 n}}}>\cdots$.

$=v\left(\left(a_{0}-a\right), \lim _{n \rightarrow \infty} \frac{1}{n^{\frac{1}{n}}}\right)$

$=v\left(\left(a_{0}-a\right), 1\right)$

$\leq v\left(a-a_{0}, 1\right), \quad$ [by (IFP.11)].

Hence

$$
v\left(a^{-1} a_{0}-e, \frac{\pi^{2}}{6}\right)<v\left(\left(a-a_{0}\right), 1\right)
$$

Let $a \in I$ and $P(a)=a^{-1}$. Let us take $\epsilon=\frac{\pi^{4}}{36}>0$,

$\mu\left(P(a)-P\left(a_{0}\right), \epsilon\right)$

$=\mu\left(a^{-1}-a_{0}^{-1}, \frac{\pi^{4}}{36}\right)$

$=\mu\left(\left(a^{-1} a_{0}-i\right) a_{0}^{-1}, \frac{\pi^{4}}{36}\right)$

$\geq \mu\left(\left(a^{-1} a_{0}-i\right), \frac{\pi^{2}}{6}\right) \diamond \mu\left(a_{0}^{-1}, \frac{\pi^{2}}{6}\right)$, [by (IFPNA.2)]

$>\mu\left(\left(a-a_{0}\right), 1\right) \diamond \mu\left(a_{0}^{-1}, \frac{\pi^{2}}{6}\right)$, [by Equation 4.2]

$>\mu\left(\left(a-a_{0}\right), 1\right) \diamond \mu\left(\left(a-a_{0}\right), \frac{\pi^{2}}{6}\right)$, [by Equation 4.1]

$\geq \mu\left(\left(a-a_{0}\right), 1\right) \diamond \mu\left(\left(a-a_{0}\right), 1\right)$, since $\mu(a, \cdot)$ is a non decreasing function and $1<\frac{\pi^{2}}{6}$ and by (2.2) of Definition

2.2 .

$>\mu\left(\left(a-a_{0}\right), 1\right),[$ by (2.6) of Definition 2.4] 
$=\mu\left(\left(a-a_{0}\right), \delta\right)$.

Also, $v\left(P(a)-P\left(a_{0}\right), \epsilon\right)$

$=v\left(a^{-1}-a_{0}^{-1}, \frac{\pi^{4}}{36}\right)$

$=v\left(\left(a^{-1} a_{0}-i\right) a_{0}^{-1}, \frac{\pi^{4}}{36}\right)$

$\leq v\left(\left(a^{-1} a_{0}-i\right), \frac{\pi^{2}}{6}\right) * v\left(a_{0}^{-1}, \frac{\pi^{2}}{6}\right)$, [by (IFPNA.2)]

$<v\left(\left(a-a_{0}\right), 1\right) * v\left(a_{0}^{-1}, \frac{\pi^{2}}{6}\right)$, [by Equation 4.3]

$<v\left(\left(a-a_{0}\right), 1\right) * v\left(\left(a-a_{0}\right), \frac{\pi^{2}}{6}\right)$, [by Equation 4.1]

$\leq v\left(\left(a-a_{0}\right), 1\right) * v\left(\left(a-a_{0}\right), 1\right)$, since $v(a, \cdot)$ is a non increasing function and $1<\frac{\pi^{2}}{6}$ and by (1.2) of Definition 2.1 .

$<v\left(\left(a-a_{0}\right), 1\right)$, [by (1.6) of Definition 2.3]

$=v\left(\left(a-a_{0}\right), \delta\right)$.

Thus for every $\epsilon>0$ there exists $\delta=\frac{\epsilon}{\frac{\pi^{4}}{36}}$ such that

$$
\mu\left(P(a)-P\left(a_{0}\right), \epsilon\right) \geq \mu\left(a-a_{0}, \delta\right), v\left(P(a)-P\left(a_{0}\right), \epsilon\right) \leq v\left(a-a_{0}, \delta\right) .
$$

Hence the mapping $P$ from $a$ to $a^{-1}$ of $I$ into $I$ is strongly IFC.

Corollary 4.4. Let $I$ be the set of invertible elements of a complete IFPNA $(V, \mu, v, \diamond, *)$. Then the mapping $P$ from $a$ to $a^{-1}$ of $I$ into $I$ is strongly IFB.

Proof.

From Theorem 4.6 of [22] and Theorem 4.3 the corollary follows.

\section{Conclusion}

In this paper, we have proposed a notion of intuitionistic fuzzy pseudo normed algebra and investigated on inverse of an element in a complete IFPNA. With suitable adaptation, the notion of IFPNA deserves attention in the study of spectrum of a bounded linear operator in intuitionistic fuzzy pseudo normed linear space.

\section{Author Contributions}

All authors contributed almost equally to this work. The manuscript has been read and approved for submission by all the named authors.

\section{Conflicts of Interest}

The authors know of no conflict of interest associated with this publication and there has been no financial support for this work that could have influenced its outcome.

\section{Acknowledgement}

The authors are grateful to the referees and the Editor for their fruitful comments, valuable suggestions and careful corrections to improve this paper.

\section{References}

[1] L. A. Zadeh, Fuzzy sets, Information and control, 8(3), (1965) 338-353. 
[2] K. T. Atanassov, Intuitionistic fuzzy sets, Fuzzy Sets and Systems, 20(1), (1986) 87-96.

[3] A. K. Katsaras, Fuzzy topological vector spaces $i$, Fuzzy sets and systems, 6(1), (1981) 85-95.

[4] A. K. Katsaras, Fuzzy topological vector spaces ii, Fuzzy sets and systems, 12(2), (1984) 143-154.

[5] C. Felbin, Finite dimensional fuzzy normed linear space, Fuzzy sets and Systems, 48(2), (1992) 239-248.

[6] S. C. Cheng, J. N. Mordeson, Fuzzy linear operators and fuzzy normed linear spaces, Bulletin of the Calcutta Mathematical Society, 86, (1994) 429-436.

[7] T. Bag, S. K. Samanta, Finite dimensional fuzzy normed linear spaces, Journal of Fuzzy Mathematics, 11(3), (2003) 687-706.

[8] R. Saadati, S. Vaezpour, Some results on fuzzy banach spaces, Journal of Applied Mathematics and Computing, 17(1), (2005) 475-484.

[9] I. Goleţ, On generalized fuzzy normed spaces and coincidence point theorems, Fuzzy sets and Systems, 161(8), (2010) 1138-1144.

[10] B. Dinda, T. K. Samanta, Intuitionistic fuzzy continuity and uniform convergence, International Journal of Open Problems in Computer Science and Mathematics, 3(1), (2010) 8-26.

[11] R. Saadati, J. H. Park, On the intuitionistic fuzzy topological spaces, Chaos, Solitons \& Fractals, 27(2), (2006) 331-344.

[12] I. Sadeqi, F. S. Kia, Fuzzy normed linear space and its topological structure, Chaos, Solitons \& Fractals, 40(5), (2009) 2576-2589.

[13] B. Dinda, T. K. Samanta, I. H. Jebril, Fuzzy anti-norm and fuzzy $\alpha$-anti-convergence, Demonstratio Mathematica, 45(4), (2012) 739-754.

[14] A. K. Mirmostafaee, Perturbation of generalized derivations in fuzzy menger normed algebras, Fuzzy sets and systems, 195, (2012) 109-117.

[15] T. Bınzar, F. Pater, S. Nadaban, On fuzzy normed algebras, Journal of Nonlinear Sciences and Applications, 9(9), (2016) 5488-5496.

[16] N. Konwar, P. Debnath, Intuitionistic fuzzy n-normed algebra and continuous product, Proyecciones (Antofagasta), 37(1), (2018) 68-83.

[17] J. R. Kider, A. H. Ali, Properties of a complete fuzzy normed algebra, Baghdad Science Journal, 16(2), (2019) 382-388.

[18] S. Nădăban, Fuzzy pseudo-norms and fuzzy f-spaces, Fuzzy Sets and Systems, 282, (2016) 99-114.

[19] T. Bag, S. K. Samanta, Fuzzy bounded linear operators, Fuzzy sets and Systems, 151(3), (2005) pp. 513547.

[20] B. Dinda, S. K. Ghosh, T. K. Samanta, Intuitionistic fuzzy pseudo-normed linear spaces, New Mathematics and Natural Computation, 15(1), (2019) 113-127.

[21] B. Dinda, S. K. Ghosh, T. K. Samanta, On w-convergence and s-convergence in intuitionistic fuzzy pseudo normed spaces, New Mathematics and Natural Computation, 17(3), (2021) 623-632. 
[22] B. Dinda, S. K. Ghosh, T. K. Samanta, Relations on continuities and boundedness in intuitionistic fuzzy pseudo normed linear spaces, arXiv preprint, arXiv:2102.06519, 2021.

[23] B. Dinda, S. K. Ghosh, T. K. Samanta, An introduction to spectral theory of bounded linear operators in intuitionistic fuzzy pseudo normed linear space, Sahand Communications in Mathematical Analysis, (2021) 12 pages (accepted), doi:10.22130/SCMA.2021.531698.942.

[24] B. Schweizer, A. Sklar, Statistical metric spaces., Pacific Journal of Mathematics, 10(1), (1960) 313 - 334.

[25] M. M. Gupta, J. Qi, Theory of t-norms and fuzzy inference methods, Fuzzy sets and systems, 40(3), (1991) 431-450. 